\title{
Content Analysis in Marketing Strategy: Applications of Hart's Theory of Word Choice and Verbal Tone: An Abstract
}

\author{
Emily Treen and Christine Pitt
}

\begin{abstract}
The amount of data from which organizational and consumer insights can be drawn is vast and increasing. Yet, the type of data that is becoming more rampant, for example, from social media, is changing to include text, images, and video. Consequently, new methods and techniques for analysis are emerging and producing fruitful insights. Yet, marketing practitioners and researchers still focus on traditional analyses from experimental or survey techniques. This paper breaks down content analysis into its theoretical underpinnings in order to shed light on its foundation as it pertains to marketing theory and practice. We draw upon Hart's theory of word choice and verbal tone to show how content analysis can be used in a variety of marketing research and strategy phenomena, both at the individual and organizational levels. Aligning with Hart's dimensions and calculated variables, which make up the theoretical foundation of DICTION, we present a number of applicable areas in marketing where content analysis can provide exceptional results. The theoretical framework proposed in this paper underscores the potential for content analysis as a viable marketing research and strategy tool for organizations.
\end{abstract}

\author{
E. Treen $(\bowtie)$ \\ Simon Fraser University, Vancouver, Canada \\ e-mail: ert1@sfu.ca \\ C. Pitt \\ Royal Institute of Technology, Stockholm, Sweden \\ e-mail: christinepitt91@gmail.com
}

